\title{
BMJ Open Effectiveness, safety and costs of orphan drugs: an evidence-based review
}

\author{
Igho J Onakpoya, ${ }^{1}$ Elizabeth A Spencer, ${ }^{1}$ Matthew J Thompson, ${ }^{2}$ Carl J Heneghan ${ }^{1}$
}

To cite: Onakpoya IJ, Spencer EA, Thompson MJ, et al. Effectiveness, safety and costs of orphan drugs: an evidence-based review. BMJ Open 2015;5:e007199. doi:10.1136/bmjopen-2014007199

- Prepublication history and additional material is available. To view please visit the journal (http://dx.doi.org/ 10.1136/bmjopen-2014007199).

Received 14 November 2014 Revised 13 May 2015 Accepted 15 May 2015

CrossMark

\footnotetext{
${ }^{1}$ Nuffield Department of Primary Care Health Sciences, University of Oxford, Centre for EvidenceBased Medicine, Oxford, UK ${ }^{2}$ Department of Family Medicine, University of Washington, Seattle, USA
}

Correspondence to Dr Igho J Onakpoya; igho.onakpoya@phc.ox.ac.uk

\section{ABSTRACT}

Introduction: Several orphan drugs have been approved by the European Medicines Agency (EMA) over the past two decades. However, the drugs are expensive, and in some instances, the evidence for effectiveness is not convincing at the time of regulatory approval. Our objective was to evaluate the clinical effectiveness of orphan drugs that have been granted marketing licenses in Europe, determine the annual costs of each drug, compare the costs of branded orphan drugs against their generic equivalents, and explore any relationships between orphan drug disease prevalence and annual costs.

Methods: We searched the EMA database to identify orphan drugs granted marketing authorisation up to April 2014. Electronic searches were also conducted in PubMed, EMBASE and Google Scholar, to assess data on effectiveness, safety and annual costs. 2 reviewers independently evaluated the levels and quality of evidence, and extracted data.

Results: We identified 74 orphan drugs, with 54 $(73 \%)$ demonstrating moderate quality of evidence. $85 \%$ showed significant clinical effects, but serious adverse events were reported in $86.5 \%$. Their annual costs were between $£ 726$ and $£ 378000$. There was a significant inverse relationship between disease prevalence and annual costs $(p=0.01)$; this was largely due to the influence of the ultra-orphan diseases. We could not determine whether the balance between effectiveness and safety influenced annual costs. For 10 drugs where generic alternatives were available, the branded drugs were 1.4 to 82000 times more expensive.

Conclusions: The available evidence suggests that there is inconsistency in the quality of evidence of approved orphan drugs, and there is no clear mechanism for determining their prices. In some cases, far cheaper generic agents appear to be available. A more robust, transparent and standard mechanism for determining annual costs is imperative.

\section{INTRODUCTION}

Orphan drugs are therapeutic agents designed for the management of orphan diseases ${ }^{1}$ which are defined as medical conditions with a low prevalence. In the USA, orphan diseases are defined as those affecting approximately 1 in 1500 persons, ${ }^{2}$ while

\section{Strengths and limitations of this study}

- We employed a robust strategy to search for the best quality evidence for effectiveness.

- We used standardised methods to rate the quality and level of evidence for each orphan drug.

- We also used reliable data to document the prevalence of each orphan disease and identify the annual cost of each orphan drug.

- Because we could not document the research and development costs associated with the approval of each orphan drug, the influence of this variable on their annual costs could not be ascertained.

- The inconsistencies in the quality of evidence for some orphan drugs limit the conclusions that could be drawn regarding their effectiveness and safety.

in Europe they are defined as those affecting 5 in $10000 .^{23}$ In Europe, ultra-orphan designations are used to specify rare diseases with prevalence of less than 1 in $50000 .^{24}$

Because low patient volumes make it unfavourable for commercial investment in orphan drug research, drug regulatory authorities have incentives in place to encourage their development and manufacture by pharmaceutical companies. In the USA, these include exclusive licensing to market such drugs for 7 years, faster assessment procedures, and tax incentives. ${ }^{1}$ In Europe, incentives include exclusive licensing for 10 years, reduction in the fees paid for regulatory activities, and provision of scientific advice by drug regulatory bodies. ${ }^{1} 5$ Despite these incentives, the costs of orphan drugs are still high, especially when generic versions of the same agent are available. ${ }^{6}$ In the UK, for example, some funders of care under the National Health Service (NHS) have refused to fund orphan drugs because they are not considered cost-effective, thereby denying patients access to potentially useful drug interventions. ${ }^{7}$ Factors that reportedly influence their price setting include research and development (R \& D) 
costs, clinical effectiveness, drug quality and disease prevalence. $^{8} 9$ However, the effectiveness of some orphan drugs has not been clearly demonstrated, and the evidence regarding their safety is often sparse at the time of regulatory approval. ${ }^{10}$

A previous systematic review of 11 orphan drugs approved in the Netherlands concluded that there is scarcity of information on the cost-effectiveness of the drugs. ${ }^{11}$ A more recent systematic review of orphan drug legislation in Europe also advocated for more stringent approval criteria for evaluating the clinical and costeffectiveness of orphan drugs. ${ }^{12}$ Another systematic review of orphan drugs used in cancers showed that they have varying levels in quality of evidence and dearth of information on economic value. ${ }^{13}$ While the first two reviews did not evaluate the quality of the evidence, the third review focused only on drugs marketed in the USA.

The objective of this review was to evaluate the effectiveness and safety of all orphan drugs that have been granted marketing licenses in Europe, determine the annual costs of each drug based on UK estimates, compare the costs of branded orphan drugs against their generic equivalents, and explore the relationship between prevalence of orphan disease and annual costs.

\section{METHODS}

We searched the European Medicines Agency (EMA) database for orphan drugs and their approved indications using the search modality 'http://www.ema.europa.eu/ ema/ $>$ find medicine $>$ human medicines $>$ Browse by type $>$ Orphan medicine: "Include authorised medicine, withdrawn post-approval and suspended" >SUBMIT'. Orphan drugs granted marketing authorisation were identified up to 30 April 2014. Drugs that have been designated 'orphan status', but have not received EMA marketing authorisation, were excluded. The EMA Orphanet Report Series (April 2014) ${ }^{14}$ was also assessed to verify approvals of the identified drug.

For each identified drug, electronic searches were then conducted in the following databases: PubMed, EMBASE, the Clinical Trials database and National Electronic Library for Medicines. The search terms used included: "orphan drug name" AND "meta-analysis", systematic review", "randomised", "clinical trial", "placebo", "follow-up study", "retrospective studies", "case-series", "cohort", "follow-up studies", "adverse events". The prevalence of orphan diseases was documented using the reference values reported in the Orphanet Report Series Bibliographic data, ${ }^{15}$ and the Orphanet portal for rare diseases and orphan drugs. ${ }^{16}$

We estimated the annual average cost in the UK for each orphan drug. Costs of orphan drug regimens might vary according to the individual patient's needs including body size, disease progression, or complications of disease. However, we did not have information on these factors, and so we used the most recent data to estimate the average annual cost in the UK. We searched the UK Medicines Information, the National Electronic Library for Medicines, North East Treatment Advisory Group (NETAG), Scottish Medicines Consortium, and All Wales Medicines Strategy Group databases, for the most recent evidence relating to the annual costs of each drug. Where these were inadequate to compute the annual costs of a specific drug, we searched The Pharma Letter (http://www.pharmaletter.com) and PharmaTimes (http://www.pharmatimes.com) websites, and Google Scholar, for the most recent data on annual costs. The costs of treatment with drugs not used on an annual basis/duration were computed as annual costs. Where orphan drugs were approved for two or more indications, we documented the annual costs of treatment separately for each indication.

For each orphan drug identified from the EMA database, we determined the level of available evidence regarding effectiveness using the Oxford Centre for Evidence-Based Medicine (OCEBM) levels of Evidence,${ }^{17}$ which comprises four levels: level 1: systematic reviews; level 2: randomised trial or observational study with dramatic effect; non-randomised controlled cohort/follow-up study; level 3: case series, case control studies, or historically controlled studies; level 4: mechanism-based reasoning.

The quality and strength of the overall body of evidence for each orphan drug was then evaluated using a checklist adapted from the Grades of Recommendation, Assessment, Development and Evaluation (GRADE) criteria, ${ }^{18}$ which assesses the five domains: study design; consistency of evidence; directness of the evidence; precision; and reporting biases. On the basis of the quality of overall evidence, one of four possible grades could be allocated: high, moderate, low and very low. Evidence for effectiveness for each orphan drug from the highest levels of evidence for each orphan drug was prioritised using the following order: meta-analysis/systematic review $>$ RCTs $>$ non-randomised studies. If two or more systematic reviews evaluated the same orphan drug, the most recent review was included. If two or more drugs were approved for treating the same orphan disease, we determined whether the level of effectiveness was related to their annual costs. Scatter plots were used to explore the relationships between prevalence of orphan disease and annual costs. If three or more drugs were approved for the same indication, we used scatter plots to test whether there was a relationship between year of approval and the annual cost. Where systematic reviews or meta-analyses did report adverse events, we searched for adverse event publications using the evidence from systematic searches using the following priority: RCTs $>$ retrospective/follow-up/cohort $>$ case-control/caseseries. Adverse events ( $\geq$ Grade 3 ) associated with the use of such drug were documented. For drugs that had been withdrawn or suspended, we documented the reasons for such decisions by accessing the EMA European Public Assessment Reports (EPAR) for human medicines. ${ }^{19}$ 
Two reviewers (IJO and EAS) independently evaluated the level, quality and strength of the evidence, and extracted data. These were then cross-checked by two other reviewers (MJT and $\mathrm{CJH})$. Disagreements were resolved through consensus.

\section{RESULTS}

The EMA database searches identified a total of 74 approved drugs (authorised medicines or medicines withdrawn postapproval) for managing 63 orphan conditions, with approval dates ranging from 15 May 2002 to 04 April 2014 (figure 1). Twenty-nine (39\%) of these drugs are used in the management of cancers, while 24 $(32.4 \%)$ are used for inborn errors of metabolism or immune disorders. The remaining 21 drugs were approved for a variety of other orphan conditions, with pulmonary arterial hypertension accounting for 5 (23\%) of the approvals. Of the 74 drugs, 5 (6.8\%) were granted conditional approval, while 15 (20\%) were granted approval under 'exceptional' circumstances. Two drugs, Cholic acid FGK and Orphacol, both contained the same active ingredient and were approved for the same indication; however, Orphacol was granted license under 'exceptional' circumstances. Two drugs, Onsenal and Rilonacept, were withdrawn by the EMA after approval because of an unfavourable risk-to-benefit profile, and commercial reasons, respectively. Details of the approval dates, levels and quality of evidence, results of clinical effectiveness, annual costs and relevant references have been included as web appendices 1-4.

\section{Levels and quality of evidence}

Using the OCEBM criteria, 25 (33.8\%) orphan drugs had level 1 evidence, 35 (47.3\%) were level 2, and 14 (18.9\%) were level 3 (figure 2A). Basing on the GRADE criteria, the overall quality of evidence could be rated as moderate in $54(73.0 \%)$ drugs, low in $16(21.6 \%)$ and very low in $4(5.4 \%)$ (figure 2B). None of the 74 drugs showed evidence of high overall quality. Relevant references for the level and quality of evidence are included as web appendix 1 .

\section{Evidence for effectiveness}

Of the 29 drugs approved for management of cancer, 6 (20.7\%) showed evidence of significant benefits for both progression-free survival and overall survival; 7 (24.1\%) showed evidence of significant improvement only in progression-free survival, while $9(31.0 \%)$ had evidence of only significantly increasing overall survival (see web appendix 2 and table S1). Of the 24 orphan drugs approved for treating inborn errors of metabolism, 21 $(75 \%)$ showed evidence of beneficial effect on at least one outcome measure (see web appendix 2 and table S2). Of the remaining 21 drugs used for managing other orphan diseases, $20(95.2 \%)$ showed evidence of significant beneficial effects (see web appendix 2 and table S3).

\section{Evidence for safety}

Twenty-eight $(96.6 \%)$ of the orphan drugs approved for treating cancerous conditions had evidence of serious adverse events (see web appendix 2 and table S1). The most common events were bone marrow suppression $(58.6 \%)$ and hepatotoxicity $(20.7 \%)$. Adverse events with Dacogen and Evoltra were severe enough to warrant premature termination of clinical trials.

Six of 24 drugs (25\%) approved for treating inborn errors of metabolism had no evidence of serious adverse events, while another (Cholic acid) did not have any preclinical safety studies prior to approval (see web appendix 2 and table S2). The most common events involved the gastrointestinal and respiratory systems (25\% and $20.8 \%$, respectively).

Eighteen of the 21 drugs $(85.7 \%)$ approved for treating other orphan conditions had evidence of serious adverse events (see web appendix 2 and table S3). Gastrointestinal adverse events were the most common $(23.8 \%)$. Other notable adverse events included cardiotoxicity $(9.5 \%)$, metabolic abnormalities $(9.5 \%)$ and possible risk of suicide with Prialt (ziconotide).

\section{Annual costs of orphan drugs}

The annual cost ranged between $£ 726$ and £378 000 (median £31 012) (details of the annual costs and the references used for documenting the evidence are shown in web appendix 3). Twenty-four per cent of the drugs cost less than $£ 10000$ annually, $58 \%$ cost between $£ 10000$ and $£ 100000$, while $18 \%$ cost $\geq £ 100000$ annually. For cancer drugs, the range was $£ 1800$ to $£ 92000$, compared with $£ 726$ to $£ 378000$ for inborn errors of metabolism.

\section{Association between disease prevalence and annual cost of orphan drugs}

A scatter plot of prevalence against annual cost (figure 3) revealed a significant inverse relationship $(p=0.01)$. By contrast, we did not observe a significant relationship when a subarea of more frequent activity (similar range of disease prevalence and annual costs) was analysed ( $p=0.56$; figure 3 inset). A significant inverse association was also observed for the relationship between annual cost and the prevalence for 21 drugs approved for managing ultra-orphan diseases $(\mathrm{p}=0.04$; figure 4$)$. However, a scatter plot of the subset of the 53 drugs approved for orphan diseases with prevalence $>1$ per 100000 revealed a non-significant relationship $(\mathrm{p}=0.18)$. Scatter plots of prevalence against annual cost did not reveal significant relationships when cancers or inborn errors of metabolism were individually tested (data not shown).

\section{Association between clinical effectiveness and annual cost}

Because of discrepancies in outcome measures and time points for outcome measurements, we could not use scatter plots to explore associations between clinical effectiveness and annual costs. All orphan drugs approved for managing pulmonary arterial hypertension 


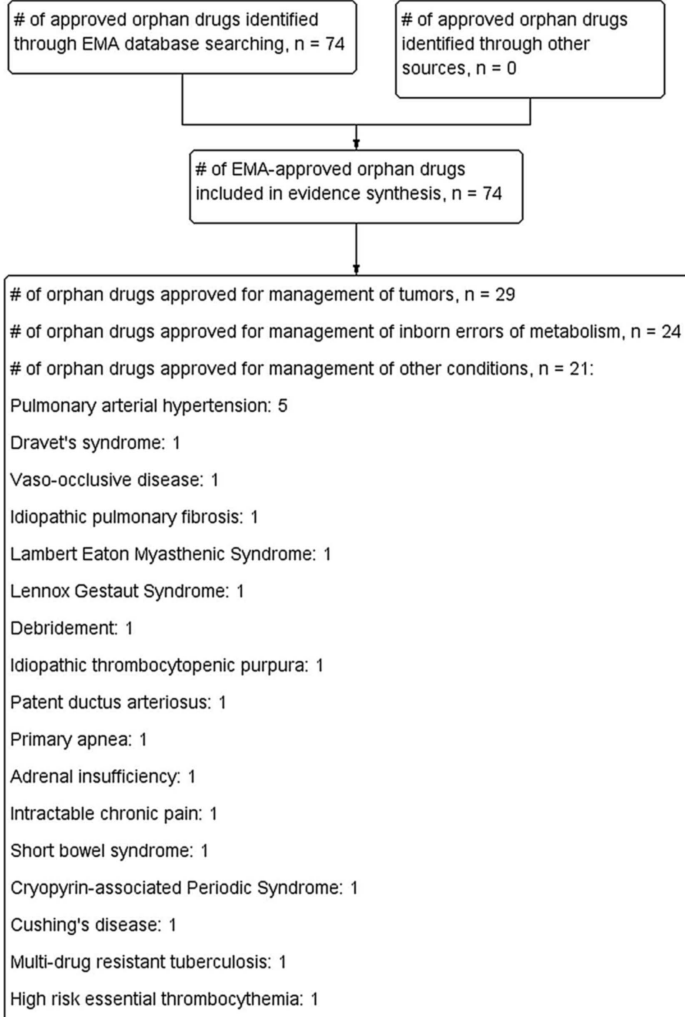

Figure 1 Flow chart showing process for inclusion of European Medicines Agency (EMA)-approved orphan drugs.

showed comparative level of effectiveness at improving 6 min work distance, and decreasing clinical worsening irrespective of annual cost (see web appendix 4). Similar findings were observed for progression-free survival and overall survival for orphan drugs approved for treating

A

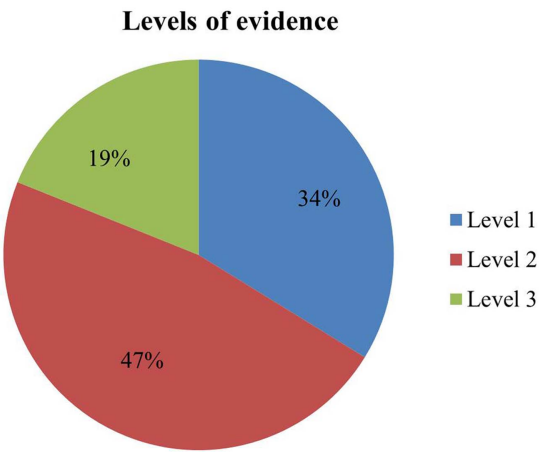

Of the 29 drugs approved for treatment of cancers, 7 (24.1\%) had Level 1 evidence, $16(55.2 \%)$ had Level 2, and $6(20.7 \%)$ were Level 3. Of the 24 drugs approved for treatment of inborn errors of metabolism, $11(45.8 \%)$ had Level 1 evidence, 7 (29.2\%) had level 2, and 6 (25.0\%) were Level 3. cancers. The annual costs of two drugs approved for treating Pseudomonas in cystic fibrosis were comparable. We could not determine whether the risk or occurrence of serious adverse events played a role in the annual costs of approved orphan drugs.

B

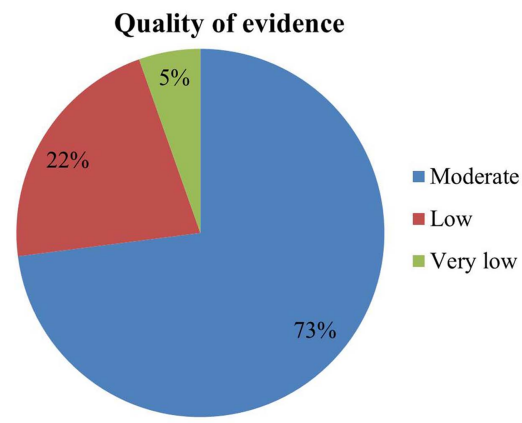

None of the approved drugs showed evidence of high overall quality. Of the 29 drugs approved for treatment of cancers, 18 (62.1\%) had moderate quality, $10(34.5 \%)$ had low quality, and $1(3.4 \%)$ was very low in quality. Of the 24 drugs approved for treatment of inborn errors of metabolism, 17 $(70.8 \%)$ had moderate quality, $4(16.7 \%)$ had low quality, and $3(12.5 \%)$ were very low in quality.

\footnotetext{
${ }^{1}$ The level of available evidence regarding effectiveness was determined using the Oxford Centre for Evidence Based Medicine (OCEBM) Levels of Evidence ${ }^{17}$ which comprises four levels: Level 1: systematic reviews; Level 2: randomized trial or observational study with dramatic effect; non-randomized controlled cohort/follow-up study; Level 3: case-series, case-control studies, or historically controlled studies; Level 4: mechanism-based reasoning. The levels of evidence could be graded down on the basis of study quality, imprecision, indirectness, inconsistency between studies, or very small absolute effect sizes; levels were graded up if there was a large or very large effect size. In the OCEBM system, a systematic review is generally better than an individual study.

${ }^{2}$ The quality and strength of the overall body of evidence for each orphan drug was evaluated using a checklist adapted from the Grades of Recommendation, Assessment, Development and Evaluation (GRADE) criteria ${ }^{18}$ which assesses the five domains: study design; consistency of evidence; directness of the evidence; precision; and reporting biases. Based on the quality of overall evidence, 1 of 4 possible grades could be allocated: high, moderate, low, and very low.
}

Figure 2 Pie charts showing the (A) levels and (B) quality of evidence of European Medicines Agency (EMA)-approved orphan drugs by proportion. 


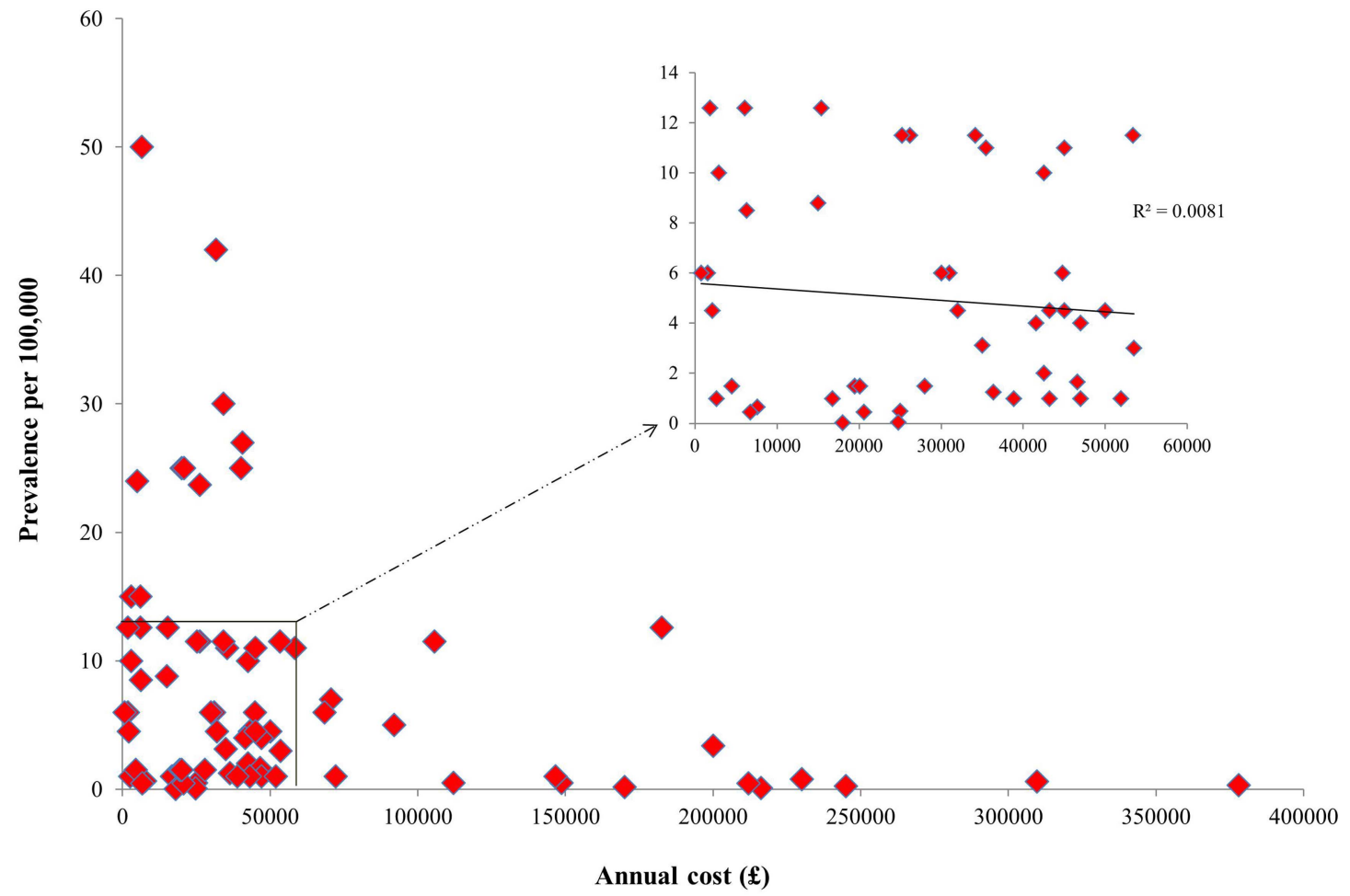

Figure 3 Scatter plot of orphan disease prevalence against annual cost.

Association between year of approval and annual cost

Two or more orphan drugs were approved by the EMA for treating seven orphan conditions (see web appendix 4). Scatter plot of annual cost against year of approval for the five drugs approved for pulmonary arterial hypertension suggested a trend towards a significant relationship for higher annual costs with more recent approvals $(\mathrm{p}=0.06)$. A significant relationship between annual cost and year of approval was observed with four drugs approved for the management of chronic myeloid leukaemia $(p=0.03)$. There was no significant relationship between annual cost and year of approval for drugs approved for treatment of acute lymphoblastic leukaemia $(p=0.38)$;

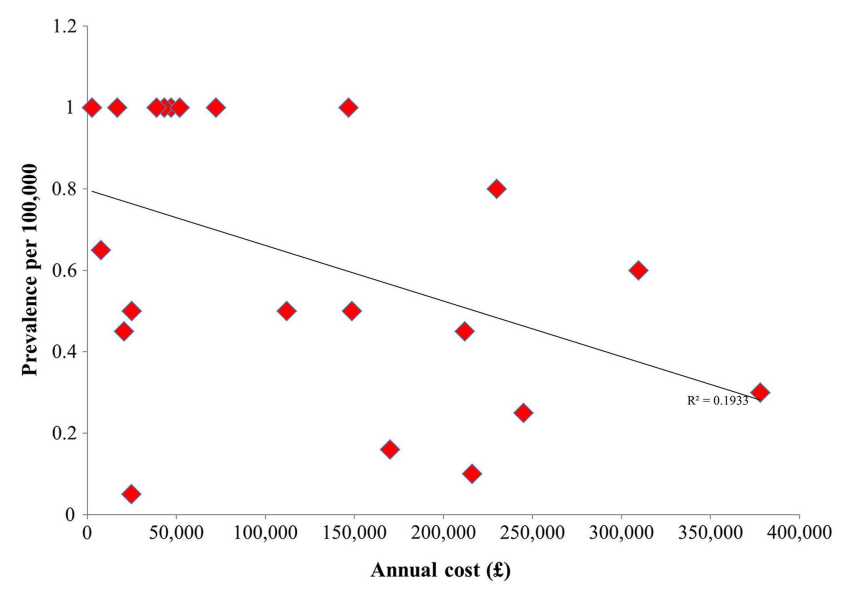

Figure 4 Scatter plot of ultra-orphan disease prevalence against annual cost. however, exclusion of the most recently approved drug of which approval was based on a historical perspective (Xaluprine) resulted in a significant relationship being observed $(\mathrm{p}=0.01)$.

\section{Branded orphan drugs versus generic or unlicensed versions}

We found 15 approved drugs with generic versions, of which data on annual cost of generic or unlicensed versions for $10(13.5 \%)$ were available. Figure 5 shows a price comparison in annual costs of branded orphan drugs compared with their unlicensed/generic equivalents. While branded mercaptopurine (Xaluprine) was only 1.4 times more costly than its generic counterpart, the branded version of intravenous ibuprofen (Pedea), used for closure of patent ductus arteriosus (PDA) in preterm infants, was 82000 times more expensive than its oral equivalent.

\section{DISCUSSION}

Main findings

Our results show that, of the 74 EMA-approved orphan drugs over a 12-year period, none has shown an overall high quality in available evidence for their effectiveness; there is a moderate level of evidence for three-quarters, while a fifth is low in evidence. Our analyses of annual drug costs revealed several interesting and potentially concerning findings. We found a significant inverse relationship between annual costs of orphan drugs and the prevalence of orphan diseases; in other words, the drugs for the most rare diseases are cheaper than those for 


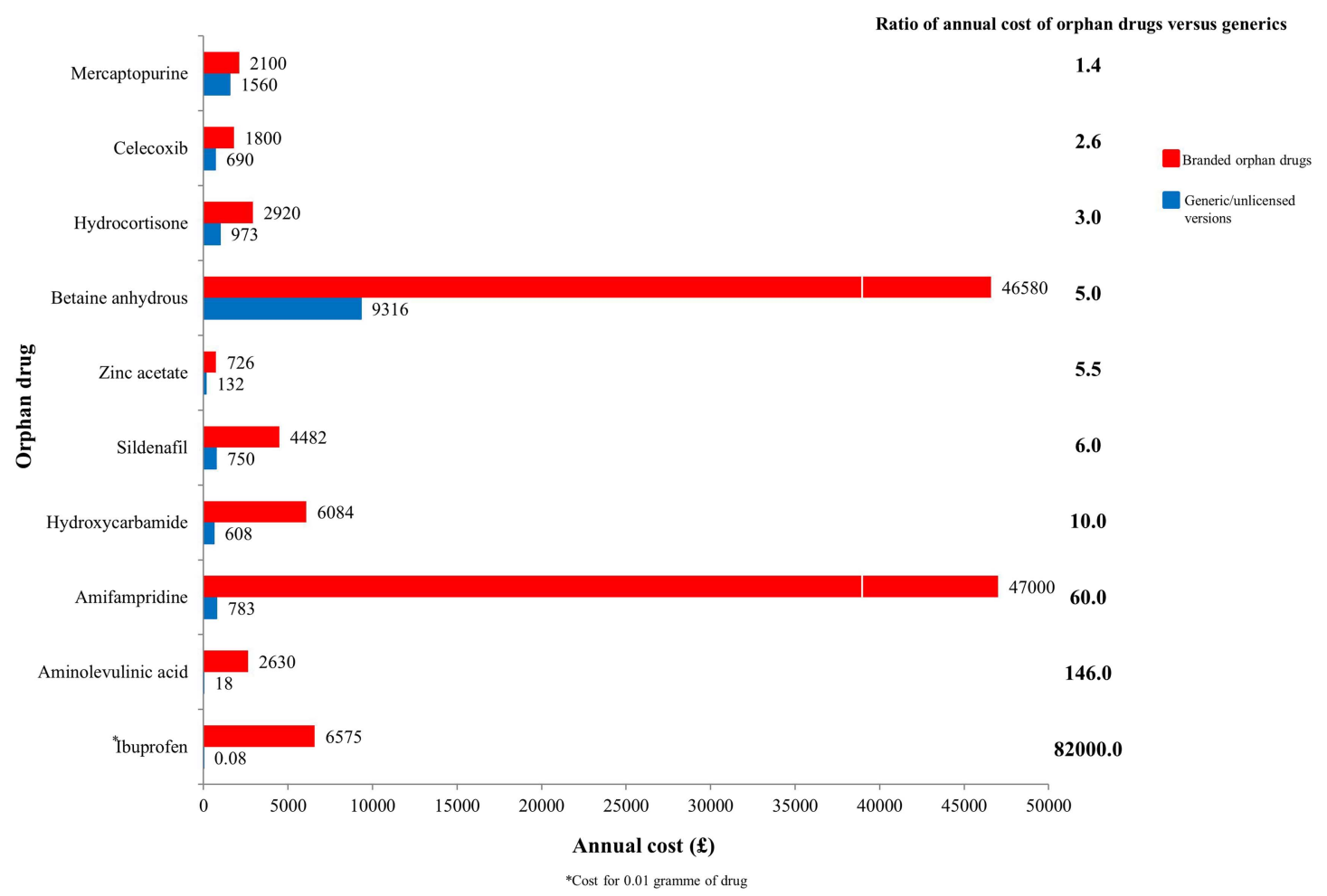

Figure 5 Price comparison of branded orphan drugs versus generic/unlicensed versions.

more common diseases, largely driven by the drug costs for the ultra-orphan diseases. We also found that drugs approved more recently cost more than those approved over the previous decade. Finally, the annual costs of the drugs did not appear to be related to their clinical effectiveness, and for the subset of the branded orphan drugs that had generic equivalents, we found several extremely large differences in cost.

While the clinical trial evidence for over two-thirds of EMA-approved orphan drugs suggests that they have clinically significant beneficial effects, the evidence for about one-fifth indicates that either they do not have clinically beneficial effects, or, more importantly, they may do harm. The results of systematic reviews for several of the drugs are also inconclusive, either because of deficiency in trial reporting or due to a lack of available clinical trials. Our inability to determine whether the level of clinical effectiveness influences annual costs for orphan diseases with two or more approved indications indicates uncertainty about how this variable determines price setting.

The findings from our review show that except for the very rare (ultra-orphan) diseases, there is no significant inverse relationship between disease prevalence and annual cost. There were inconsistencies in the strengths of correlation between year of approval and annual costs when three or more drugs used for the same orphan condition were compared. This indicates that there is no standardised process for setting the prices of orphan drugs. It is important to realise that many orphan drugs target very small patient numbers, and are for conditions with few treatment alternatives, thereby allowing their prices to be relatively higher than non-orphan drugs.

\section{Comparison with the existing literature}

The results of our review confirm a previous research report, which concluded that prevalence of orphan disease is inversely associated with annual costs for only very rare diseases. ${ }^{8}$ Our results also corroborate results from a previous review which showed that approved orphan drugs vary in the quality of evidence, ${ }^{13}$ and support the evidence from two previous reviews which suggested that there is no clarity about how clinical effectiveness is used to determine the costs of orphan drugs. ${ }^{11}{ }^{12}$ Contrasting with previous reports, our review is more systematic, and by far more comprehensive. We also determined whether year of approval has any influence in determining costs, and we compared branded versions of the orphan drugs with their unlicensed or generic versions. Our results support the findings of a qualitative analysis examining reimbursement for orphan drug prescriptions in Belgium, which suggested that more standardisation of how orphan drugs are priced is needed. ${ }^{20}$ However, we investigated all orphan drugs approved for use in Europe, and assessed the quality of evidence for effectiveness and safety.

\section{Strengths and limitations}

We employed a robust strategy to search for the best quality evidence for effectiveness, safety and annual costs of each orphan drug, and we used standardised methods to rate the quality and level of evidence for 
each identified drug. We also used reliable data to document the prevalence of each orphan disease and identify the annual cost of each orphan drug.

However, we do recognise several limitations. Because we could not document the R\&D costs associated with the approval of each orphan drug, the influence of this variable on their annual costs could not be ascertained. The heterogeneity in clinical outcomes across different diseases, discrepancies in time points for outcome measurements, and the inconsistency in the levels of outcome reporting, also limited the type of analyses and, therefore, the conclusions that could be drawn regarding the effectiveness and safety of some orphan drugs.

\section{Implications for research}

Our finding that the trial evidence for the effectiveness and safety of $65 \%$ of drugs has not been systematically reviewed suggests an urgent need for prioritising reviews for these drugs. Moreover, systematic reviews evaluating the effectiveness and safety of some EMA-approved orphan drugs are now outdated because more recent clinical trials have become available, and, consequently, such reviews need to be updated. Given the value of systematic reviews for clinicians and health policymakers, we suggest the formation of a Cochrane Collaboration review group focused on orphan drugs as one way of reducing these gaps in knowledge.

In addition, further independent and industry-funded trials are also warranted, especially for drugs that currently have poor quality and low levels of evidence. Clinical trial investigators should adequately report any suspected adverse events observed in the course of trials; and statements made in some primary studies that such events, if any, were not related to the drug in question (or not serious enough) may be premature. The importance of close postapproval monitoring cannot be overemphasised. Finally, given the inconsistencies we found in drug costs, a more detailed and transparent analysis of the relationship between $\mathrm{R} \& \mathrm{D}$ costs, and annual costs of orphan drugs, is now imperative.

\section{Implications for policy}

The use of novel therapeutic agents has resulted in improvements in clinical outcome for several orphan diseases. It is also rational that drug companies should aim to recoup investment in R\&D costs through sales and reimbursements for these drugs, even though the size of the market for such drugs is quite small. ${ }^{21}$ However, the overall benefits on clinical and economic terms need to be taken into consideration when setting prices. ${ }^{22}$ In fact, unfavourable cost-to-benefit analysis has led to the rejection of applications for approval of orphan drugs by the UK National Institute for Health and Care Excellence. ${ }^{23} 24$ Recently, NHS England threatened to stop purchasing some orphan drugs used for cancer management, unless the manufacturers reduced their prices. ${ }^{25}$ Since the costeffectiveness of orphan drugs is difficult to assess, considering opportunity costs might help in making decisions about funding the provision of orphan drugs.

The high costs of orphan drugs are reportedly due to the large amounts of funds invested in R\&D by the drug manufacturers. ${ }^{26}$ However, Light and Lexchin ${ }^{27}$ reported that drug companies spend only $1.3 \%$ of their revenue on basic research in order to discover novel molecules. In addition, the disparity in prices when these branded drugs are compared with their generic versions makes it doubtful if the quoted $\mathrm{R} \& \mathrm{D}$ costs are sufficiently robust. It is unclear in respect of the extent to which the incentives offered by the EMA and other drug regulatory authorities to encourage the development of these drugs influenced the prices of these branded drugs. For example, Pedea costs 82000 times as much as generic ibuprofen, yet, research evidence has shown that Pedea is not superior to oral ibuprofen in clinical effectiveness regarding closure of PDA. ${ }^{28}$ This raises the question of whether large national health systems, such as the NHS, should choose generic versions of orphan drugs where available, particularly given current pressure on healthcare expenditures. ${ }^{29}$ In 2013, for example, the drug company Novartis AG lost its patenting right for Glivec (imatinib) in India, largely due to its very high price compared to its generic alternative. ${ }^{30}$

The marketing authorisations granted to some orphan drugs, such as Ceplene, Firdapse and Xaluprine, were based on a historical perspective, that is, these compounds have previously been used in patient management prior to being designated orphan status. ${ }^{31}$ Therefore, in cases such as these, it does not seem plausible for drug companies to claim huge $\mathrm{R} \& \mathrm{D}$ costs because they are not new therapies. Indeed, the application for approval of Ceplene for its designation as an orphan drug was refused in Canada due to a failure of the drug company to prove that its development was innovative. ${ }^{32}$ Similarly, it would be expected that the prices of drugs for which there are alternative therapeutic options for their stated indications would be competitively based.

The postapproval withdrawal of some orphan drugs suggests that premarketing and postmarketing surveillance is not stringently assessed (or evaluated) during the process of clinical trial and drug approval. For example, Onsenal (celecoxib) was also withdrawn 8 years postapproval after it was discovered that the risks outweighed its benefits in its use for managing familial adenomatous polyposis. ${ }^{33}$ Thelin (sitaxentan) lost its orphan status (and was withdrawn from the market) due to fatal hepatotoxicity; ${ }^{34}$ clinical trial results had shown that the drug had adverse effects on the liver, but these were not considered serious enough to prevent its approval. The serious adverse events associated with some approved drugs lend credence to the view that safety does not appear to be a factor when determining the costs of the drugs.

\section{CONCLUSION}

There is inconsistency in the level and quality of evidence for approved orphan drugs. While some orphan 
drugs have demonstrated evidence of significant benefits, evidence of effectiveness is lacking for several others, and some are associated with serious unwanted adverse effects. The available evidence suggests that, except for the ultra-orphan diseases, the annual costs of orphan drugs approved in Europe are not influenced by disease prevalence. There is inadequate data to determine whether clinical effectiveness influences the price setting of orphan drugs. Further research into the effectiveness and safety of orphan drugs is required, and a standard, transparent and robust mechanism for determining their prices should be a priority.

Contributors IJO was involved with literature searches, data extraction, data analysis and interpretation, and co-drafting of the review. EAS was involved with literature searches, data extraction, data analysis and co-drafting of the review. MJT and CJH were involved with data analysis and interpretation, and co-drafting of the review.

Funding This research was funded by the National Institute for Health Research (NIHR) School for Primary Care Research (SPCR) (grant number 234).

Disclaimer This article presents independent research funded by the National Institute for Health Research (NIHR).

Competing interests CJH receives payment for running educational courses at the University of Oxford and University of Oxford ISIS consulting services for external teaching and training. He also receives royalties for books (Evidence Based Toolkit series by Blackwell BMJ Books).

Provenance and peer review Not commissioned; externally peer reviewed.

Data sharing statement No additional data are available.

Open Access This is an Open Access article distributed in accordance with the terms of the Creative Commons Attribution (CC BY 4.0) license, which permits others to distribute, remix, adapt and build upon this work, for commercial use, provided the original work is properly cited. See: http:// creativecommons.org/licenses/by/4.0/

\section{REFERENCES}

1. Dear JW, Lilitkarntakul P, Webb DJ. Are rare diseases still orphans or happily adopted? The challenges of developing and using orphan medicinal products. Br J Clin Pharmacol 2006;623:264-71.

2. Sharma A, Jacob A, Tandon M, et al. Orphan drug: development trends and strategies. J Pharm Bioallied Sci 2010;2:290-9.

3. European Medicines Agency (EMA). Orphan drugs and rare diseases at a glance. http://www.ema.europa.eu/docs/en_GB/ document_library/Other/2010/01/WC500069805.pdf

4. National Institute for Clinical Excellence. NICE citizens council report ultra orphan drugs. London: NICE, 2004. http://www.nice.org.uk/ proxy/?sourceUrl=http://www.nice.org.uk/niceMedia/pdf/ boardmeeting/brdjan05item4.pdf (accessed 7 May 2015).

5. Hughes DA, Tunnage B, Yeo ST. Drugs for exceptionally rare diseases: do they deserve special status for funding? QJM 2005;98:829-36.

6. Simoens $\mathrm{S}$, Cassiman D, Picavet E, et al. Are some orphan drugs for rare diseases too expensive? A study of purchase versus compounding costs. Drugs Ther Perspect 2011;27:24-6.

7. Hawkes N, Cohen D. What makes an orphan drug? BMJ 2010;341: c6459.

8. Scherer FM. The pharmaceutical industry-price and progress. N Engl J Med 2004;351:927-32.

9. van Ekdom L. Price setting orphan drugs. Identifying the influential factors on the price setting of Orphan Drugs [MSc thesis]. 2006. http://www.ppge.ufrgs.br/ats/disciplinas/1/vanekdom-2006.pdf (accessed 20 Mar 2013).
10. McCabe C, Claxton K, Tsuchiya A. Orphan drugs and the NHS: should we value rarity? BMJ 2005;331:1016-19.

11. Kanters TA, de Sonneville-Koedoot C, Redekop WK, et al. Systematic review of available evidence on 11 high-priced inpatient orphan drugs. Orphanet J Rare Dis 2013;8:124. .

12. Joppi R, Bertele' V, Garattini S. Orphan drugs, orphan diseases. The first decade of orphan drug legislation in the EU. Eur J Clin Pharmacol 2013;69:1009-24.

13. Cheng MM, Ramsey SD, Devine EB, et al. Systematic review of comparative effectiveness data for oncology orphan drugs. $A m \mathrm{~J}$ Manag Care 2012;18:47-62.

14. Orphanet Report Series. Orphan drug collection. List of medicinal products for rare diseases in Europe. April 2014. http://www.orpha. net/orphacom/cahiers/docs/GB/list_of_orphan_drugs_in_europe.pdf (accessed 24 Apr 2014).

15. Orphanet report series - prevalence of rare diseases: bibliographic data. http://www.orpha.net/orphacom/cahiers/docs/GB/Prevalence of rare diseases_by_alphabetical_list.pdf

16. Orphanet: The portal for rare diseases and orphan drugs. http:// www.orpha.net/consor/cgi-bin/index.php?Ing=EN

17. OCEBM Levels of Evidence Working Group*. "The Oxford 2011 Levels of Evidence". Oxford Centre for Evidence-Based Medicine. $\mathrm{http}: / /$ www.cebm.net/index.aspx?o=5653

18. Guyatt GH, Oxman AD, Vist GE, et al. GRADE Working Group. GRADE: an emerging consensus on rating quality of evidence and strength of recommendations. BMJ 2008;336:924-6.

19. European Medicines Agency. European public assessment reports. http://www.ema.europa.eu/ema/index.jsp?curl=pages/medicines/ landing/epar_search.jsp\&mid=WCOb01ac058001d125

20. Denis A, Mergaert L, Fostier C, et al. Critical assessment of Belgian reimbursement dossiers of orphan drugs. Pharmacoeconomics 2011;29:883-93.

21. Drummond MF, Wilson DA, Kanavos $\mathrm{P}$, et al. Assessing the economic challenges posed by orphan drugs. Int J Technol Assess Health Care 2007;23:36-42.

22. Picavet E, Morel T, Cassiman D, et al. Shining a light in the black box of orphan drug pricing. Orphanet J Rare Dis 2014;9:62.

23. UK cost agency says "no" to Novartis blood cancer drug Jakavi. http://www.reuters.com/article/2013/02/13/us-novartis-britain-jakaviidUSBRE91C00Y20130213 (accessed 1 Mar 2013).

24. Pharmafile. NICE rejects Pfizer blood cancer drug. 16/07/2013. http://www.pharmafile.com/news/180127/nice-rejects-pfizer-bloodcancer-drug (accessed 25 Apr 2014).

25. BBC News Health. NHS England push to rein in cancer drug prices. http://www.bbc.co.uk/news/health-28938022 (accessed 28 Aug 2014).

26. Simoens S. Pricing and reimbursement of orphan drugs: the need for more transparency. Orphanet $J$ Rare Dis 2011;6:42.

27. Light DW, Lexchin J. Foreign free riders and the high price of US medicines. BMJ 2005;331:958-60.

28. Neumann R, Schulzke SM, Bührer C. Oral ibuprofen versus intravenous ibuprofen or intravenous indomethacin for the treatment of patent ductus arteriosus in preterm infants: a systematic review and meta-analysis. Neonatology 2012;102:9-15.

29. Gov.Uk. Policy. Making NHS more efficient and less bureaucratic. 25 March 2013. https://www.gov.uk/government/policies/making-thenhs-more-efficient-and-less-bureaucratic (accessed 21 May 2013)

30. BBC News. Novartis: India rejects patent plea for cancer drug Glivec. http://www.bbc.co.uk/news/business-21991179 (accessed 1 Apr 2013).

31. Wirtz PW, van Dijk JG, van Doorn PA, et al. The epidemiology of the Lambert-Eaton myasthenic syndrome in the Netherlands. Neurology 2004;63:397-8.

32. Epicept Corporation v. Canada (Health). 2010 FC 956,[2010] 4 F.C. R. D-9. http://reports.fja.gc.ca/eng/2010/2010fc956.pdf (accessed 3 Sep 2014).

33. European Medicines Agency. Assessment Report for Celecoxib for the reduction of the number of adenomatous intestinal polyps in familial adenomatous polyposis, as an adjunct to surgery and further endoscopic surveillance. http://www.ema.europa.eu/docs/en_GB/document_library/ Report/2011/06/WC500107627.pdf (accessed 28 Jan 2013).

34. European Medicines Agency. Thelin (sitaxentan) to be withdrawn due to cases of unpredictable serious liver injury. http://www.ema. europa.eu/docs/en_GB/document_library/Press_release/2010/12/ WC500099707.pdf (accessed 22 Feb 2013). 


\section{Correction}

Onakpoya IJ, Spencer EA, Thompson MJ, et al. Effectiveness, safety and costs of orphan drugs: an evidence-based review. BMJ Open 2015;24;5:e007199. There have been corrections to the online supplement as follows:

In Appendix 1, Orphacol has been added to the list, and the appropriate references used in the quality and level of the evidence for Orphacol and Cholic Acid FGK are:

- Cholic Acid FGK: European Medicines Agency. Assessment report. Cholic acid FGK. http://www.ema.europa.eu/docs/en_GB/document_library/EPAR_-_Public_ assessment_report/human/002081/WC500165386.pdf

- Orphacol: European Medicines Agency. Orphacol, INN-cholic acid - Annex 1. Summary of product characteristics http://www.ema.europa.eu/docs/en_GB/ document_library/EPAR__Product_Information/human/001250/WC500150993. pdf

In Appendix 2, table 2, the authors have revised the indications for Orphacol, and added 'deficiency' to the indication for Cholic Acid FGK:

Cholic Acid FGK: IEM in primary bile acid synthesis due to Sterol 27-hydroxylase deficiency.

Orphacol: IEM in primary bile acid synthesis due to 3beta-Hydroxy-delta5-C27-steroid oxidoreductase deficiency or delta4-3-Oxosteroid-5beta-reductase.

In Appendix 2, table 2, and in Appendix 3, the authors have revised the annual cost of Litak to $£ 835$ ( 1 cycle) based on correspondence with the manufacturers.

BMJ Open 2015;5:e007199corr1. doi:10.1136/bmjopen-2014-007199corr1 